\title{
STAT1 inhibitor alleviates spinal cord injury by decreasing apoptosis
}

\author{
Y.X. Wu', C.Z. Gao', K.L. Fan', L.M. Yang ${ }^{3}$ and X.F. $\mathrm{Mei}^{3}$ \\ ${ }^{1}$ Department of Spinal Surgery, Second Hospital of Shandong University, Jinan, China \\ ${ }^{2}$ Department of Emergency, \\ The Affiliated Hospital of Shandong University of Traditional Chinese Medicine, \\ Jinan, China \\ ${ }^{3}$ Department of Orthopedics, First Hospital of Liaoning Medical University, Jinzhou, \\ China \\ Corresponding author: C.Z. Gao \\ E-mail: gaochunzheng@yeah.net \\ Genet. Mol. Res. 15 (1): gmr.15017271 \\ Received July 21, 2015 \\ Accepted December 4, 2015 \\ Published March 28, 2016 \\ DOI http://dx.doi.org/10.4238/gmr.15017271
}

ABSTRACT. Spinal cord injury $(\mathrm{SCl})$ is typically caused by trauma or disease, and it severely affects patients' motor function. The relationship between signal transducers and activators of transcription-1 (STAT1) and neuronal death after cerebral focal ischemia has been comprehensively studied, but its role in SCI remains largely unknown. This study investigated the protective effect of an STAT1 inhibitor on SCI. Thirty SD rats were SCIinduced and were then randomly divided into two groups ( $N=15$ each), either receiving STAT1 or the STAT1 inhibitor S1491 by intraperitoneal injection. The motor dysfunction of the rats was evaluated by behavioral scores, followed by the examination of $\mathrm{SCl}$ by hematoxylin and eosin staining. Apoptosis was also detected by Western blot and terminal deoxynucleotidyl transferase-mediated dUTP nick-end-labeling (TUNEL) assay. The motor functions of rats receiving STAT1 did not score as well as the STAT1 inhibitor group $(P<0.01)$. Further assays showed remarkable improvements in pathological damage to spinal code tissue in STAT1 inhibitor-treated rats, along with lower Bax and higher $\mathrm{Bcl}-2$ expression. 
The STAT1 inhibitor also suppressed the occurrence of TUNEL-positive cells compared to the STAT1-treated group. In summary, we suggest that the STAT1 inhibitor alleviates SCI by decreasing apoptosis.

Key words: Signal transducers and activators of transcription-1 (STAT1); Spinal cord injury; Apoptosis

\section{INTRODUCTION}

Spinal cord injury $(\mathrm{SCl})$, which can be caused by both primary and secondary factors, severely compromises the quality of life of patients. In primary $\mathrm{SCl}$, irreversible damage occurs within a short time, whereas secondary $\mathrm{SCl}$ involves a series of pathological alterations including focal tissue ischemia, edema, and inflammation (Cao and Dong, 2013; Hua et al., 2013). Signal transducers and activators of transcription-1 (STAT1) has been known to regulate the gene transcription of various cytokines, which further activates the Janus kinase/STAT1 pathway by phosphorylation and nuclear translocation, thus potentiating its target gene transcription (Oyinbo, 2011; Laurence et al., 2012; Aittomäki and Pesu, 2014).

Previous reports have shown the significance of post-SCI upregulation of inflammatory

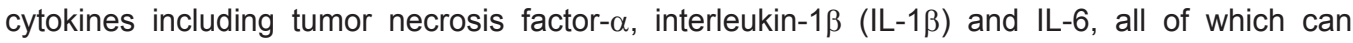
cause secondary injuries. In fact, in some studies, researchers have attempted to alleviate $\mathrm{SCl}$ by suppressing expression of these genes (O'Shea et al., 2013; Vawda and Fehlings, 2013). Apoptosis, one process of many in programmed cell death, is a critical step in $\mathrm{SCl}$ formation and development. Current anti-apoptotic drugs depress the release of mitochondrial cytochrome $\mathrm{C}$ by suppressing cerebral excitatory toxicity. The downregulation of cytochrome $\mathrm{C}$ arrests the expression of apoptosis-related genes, including caspase 23 and Bax, and potentiates anti-apoptotic proteins such as Bcl-2 by a cascade reaction, suppressing injury and apoptosis of spinal cord neural cells and alleviating SCl (Vahedi et al., 2012; Schmitt et al., 2015). STAT1 functions as an important transcription factor in the cellular response of cytokines; however, its potential role in and effects on $\mathrm{SCl}$ remain unknown. The purpose of this study was to further investigate the role of STAT1 in SCI.

\section{MATERIAL AND METHODS}

\section{SCI model generation}

The SCI model was prepared in 30 male SD rats as previously described (Lu et al., 2014). After general anesthesia and skin sterilization, an area of $9 \mathrm{~mm}^{2}$ around the T10 spinous process was exposed. The process was clipped by a surgical clamp for $3 \mathrm{~s}$. The occurrence of spastic tail movement, retraction of lower body and limbs, followed by the paralysis of lower limbs suggests the successful generation of $\mathrm{SCl}$ model. After surgery to induce $\mathrm{SCl}$, the rats were randomly divided into two groups ( $\mathrm{N}=15$ each): STAT1 and STAT1 inhibitor S1491 group. Rats were intraperitoneal injected with STAT1 or S1491 at a dose of $0.1 \mu \mathrm{L} / \mathrm{g}$ of body weight each day for a total of 30 consecutive days. All procedures were approved by the Animal Ethics Committee of Second Hospital of Shandong University.

\section{Motor dysfunction grading}

All rats received an acclimation (about 5-10 $\mathrm{min}$ ) before the test. The observer then 
evaluated the voluntary movement of rats and marked a score up to 9 points. In brief, normal movement with stable body was denoted as 9 points. The incoordination between fore- and hindlimbs during normal movement was marked as 5-8 points. Slow movement using only hind limbs to support the body was denoted as 3-4 points. Movement only of the ankles and no other body parts represented 0-2 points.

\section{Hematoxylin and eosin (H\&E) staining}

Spinal cord samples were collected and fixed in $4 \%$ formalin overnight. After dehydration using an ethanol gradient ( $70 \%$ for $3 \mathrm{~h}, 80 \%$ for $3 \mathrm{~h}, 95 \%$ for $2 \mathrm{~h}$, and $100 \%$ for $1.5 \mathrm{~h}, 2$ times each), tissue samples were immersed in xylene $(1.5 \mathrm{~h}, 2$ changes) followed by paraffin incubation $(1 \mathrm{~h}$ $+2 \mathrm{~h}$ at $60^{\circ} \mathrm{C}$ ). The sample was then embedded in paraffin and cut into 3- $\mu \mathrm{m}$ thick slices, which were mounted onto glass slides. Before staining, sections were dewaxed in xylene, followed by re-hydration in an ethanol gradient (xylene, followed by 100, 95, 90, 85, and 80\% ethanol, 10 min each). Tissues slides were stained in hematoxylin for $1 \mathrm{~min}$ and washed with water. The eosin dye was then added for $10 \mathrm{~s}$ counter-staining. After stopping, dehydration and coverslip mounting were performed. High-magnification (20X) images were captured from the central region of the sample, and were judged by three independent observers.

\section{Terminal deoxynucleotidyl transferase-mediated dUTP nick-end-labeling (TUNEL)}

Tissue slides were prepared as described in the previous section. After dewaxing and washing with PBS, antigen retrieval was performed using citric acid buffer $(\mathrm{pH} 6)$ and heating. After cooling down, endogenous peroxidase activity was quenched by $3 \% \mathrm{H}_{2} \mathrm{O}_{2}$ in methanol for 5 min at $22^{\circ} \mathrm{C}$. Terminal deoxynucleotidyl transferase (TdT) enzyme buffer (Santa Cruz, USA) was then applied for 5-min pre-incubation, followed by addition of the TdT reaction reagent for 1-h incubation at $37^{\circ} \mathrm{C}$. The streptavidin-horseradish peroxidase working solution (1:500 dilution) was then added for 30-min incubation. After rinsing in PBS (5 min, 3 times), anti-digoxigenin antibody conjugated with peroxidase (Zhongshan Biotech, Beijing, China) was applied for 30-min incubation at room temperature. Fresh 3,3'-diaminobenzidine substrates (0.05\%) (Zhongshan Biotech) were then added for 10-min development. After rinsing and mounting, stained images were captured under an inverted light microscope.

\section{Western blotting}

Total proteins were extracted by a protein purification kit (Beyotime, China) and quantified by BCA solution (1:50 dilution). All proteins were made to equal concentrations and $0.2 \mathrm{X}$ bromophenol blue was added. Protein samples were separated by SDS-PAGE (120 V) and transferred to PVDF membrane at $250 \mathrm{~mA}$. The membrane was rinsed in 1X TBS-T and blocked with $5 \%$ skim milk powder. After washing with 1X TBS-T, primary antibody (rabbit anti-Bax or anti-Bcl-2; Santa Cruz) at 1:100 dilution was added to the membrane and incubated overnight. After washing with $1 \mathrm{X}$ TBS-T, secondary goat anti-rabbit antibody at 1:100 dilution (Santa Cruz) was added followed by enhanced chemiluminescence development. After exposure, the intensity of bands was detected by a Molecular Imager ${ }^{\circledR}$ ChemiDoc $^{\mathrm{TM}}$ XRS System (Bio-Rad Laboratories, Hercules, CA, USA). The gray value of bands was analyzed by the Image Lab 2.0 software (Bio-Rad Laboratories) by normalization against the GAPDH housekeeping gene. 


\section{Statistical analysis}

The SPSS 11.0 software package was used to process all collected data. The Student $t$-test was used to compare means between groups. Pearson analysis was employed to reveal correlation between parameters. Chi-square analysis was used to compare non-parametric data. Statistical significance was defined as $\mathrm{P}<0.05$.

\section{RESULTS}

\section{STAT1 inhibitor S1491 improved motor function after SCI}

Two different groups of rats were treated with either STAT1 or S1491, which is a STAT1 inhibitor, 28 days after SCl-induction surgery. The motor behavior of the rats was observed and scored and the results are shown in Figure 1. The treatment of rats with $\mathrm{S} 1491$ significantly improved the motor score compared to the STAT1 treatment group $(P<0.01)$.

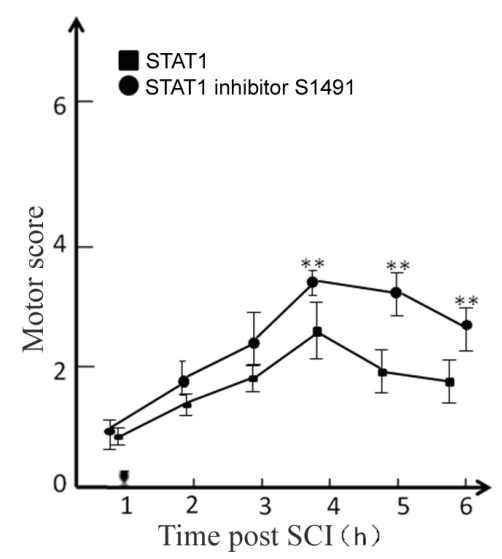

Figure 1. Animal motor score post-spinal cord injury $(\mathrm{SCl})$ induction. Scores are shown for animals treated with STAT1 (STAT1 treatment) and S1491 (STAT1 inhibitor). ${ }^{* *} \mathrm{P}<0.01$ compared to STAT1 treatment group.

\section{Effects of S1491 on SCI}

In evaluating motor function, we found that the application of the STAT1 inhibitor significantly improved scores compared to STAT1 treatment. Therefore, we hypothesized that treatment with the STAT1 inhibitor would also improve SCI. This was supported by H\&E staining of spinal cord tissue, where several neurons were found with dispersed vacuoles in the STAT1 treatment (Figure 2A). However, in STAT1 inhibitor-treated animals, we found fewer neurons containing tissue vacuoles (Figure 2B). Therefore, our results show that treatment with an STAT1 inhibitor could alleviate SCI.

\section{Decreased apoptosis after treatment with STAT1 inhibitor (S1491)}

The STAT1 inhibitor alleviated SCI but the mechanisms by which this occurs is unknown. We next examined if the STAT1 inhibitor S1491 might mediate apoptosis. To do this, we took the total protein from spinal cord tissues and quantified two apoptotic proteins (Bax and Bcl-2) by 
western blot. We found that Bax was downregulated and $\mathrm{Bcl}-2$ was upregulated in the STAT1 inhibitor-treated group compared to STAT1 treatment $(P<0.05$; Figure $3 A$ and $B)$. Upregulation of the anti-apoptotic protein $\mathrm{Bcl}-2$ and downregulation of the pro-apoptotic protein Bax support the role of $\mathrm{S} 1491$ in decreasing apoptosis. We further detected apoptosis by TUNEL staining. As shown in Figure 3C, TUNEL-positive cells were found in the STAT1-treated group but not in the STAT1 inhibitor-treated group.
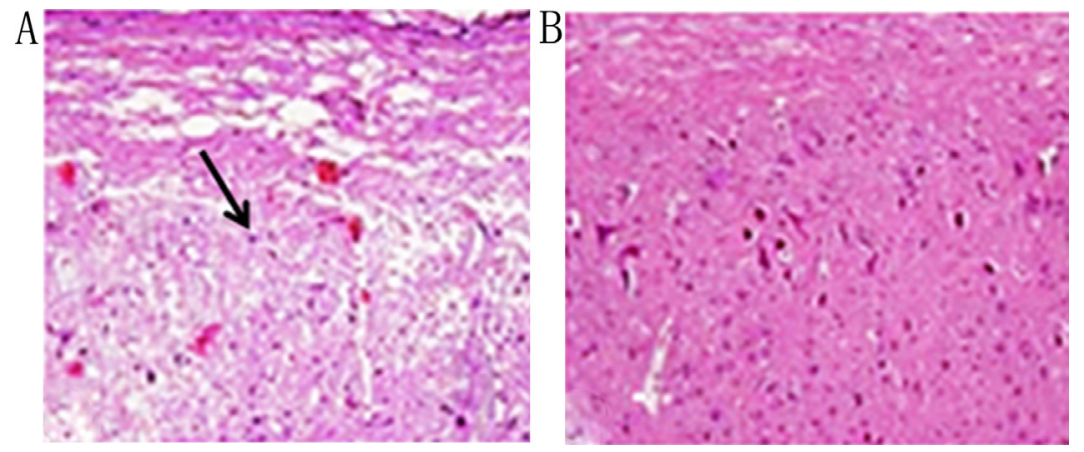

Figure 2. Spinal cord tissue morphology by H\&E staining after STAT1 treatment (A) and STAT1 inhibitor (S1491) treatment (B). Arrow shows a neuronal cell.
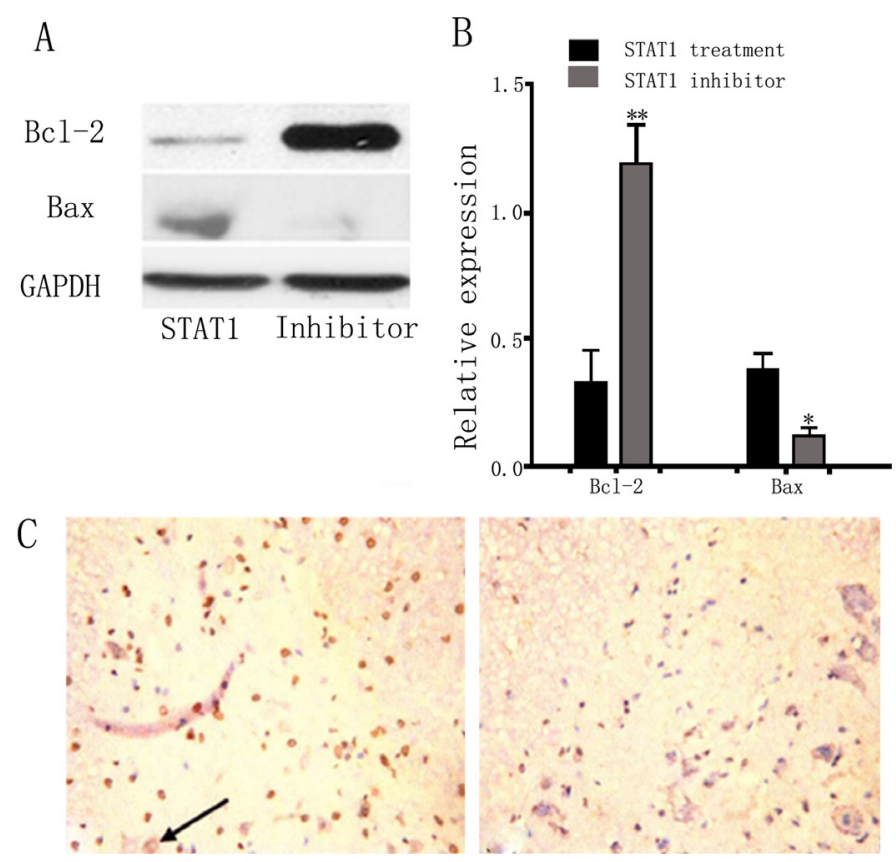

Figure 3. Cell apoptosis in spinal cord tissues. A. Western blot showing downregulation of Bax and upregulation of $\mathrm{Bcl}$-2 proteins in the STAT1 inhibitor (S1491)-treated group compared to the STAT1-treated group. GAPDH is shown as an internal control. B. Quantified results of the western blot in $(A)$ with ${ }^{* *} P<0.01$ and ${ }^{*} P<0.05$ compared to the STAT1 treatment group. C. TUNEL staining showing decreased apoptotic cells in the STAT1 inhibitor group (right) compared to the STAT1 treatment group (left). Arrow shows a TUNEL-positive neuronal cell. 
Y.X. Wu et al.

\section{DISCUSSION}

More than 10,000 people in China suffer from SCI each year, mainly due to traffic accidents, exercise and other traumatic injuries, and it leads to pathological damage and neurological dysfunction (Han et al., 2012). Physical injury is the most common reason underlying $\mathrm{SCl}$ and it results in both primary and secondary injuries, the latter of which include apoptosis of neuronal and glial cells, elevated permeability of the spinal cord barrier, and elongation of the neural reflex response (Ishii et al., 2013). The secondary inflammatory response in SCl is even more severe in the spinal cord than in cerebral damage. Various experiments and trials have been performed in animal models attempting to modify the composition of inflammatory cells in secondary injury but little progress has been made. Current research has focused on the alternation of neural stem cells after the inflammatory response but further optimization and clinical validation are required (Cao et al., 2010; Gómez-Nicola et al., 2011; Yoshimura et al., 2011).

Previous studies have reported the occurrence of apoptosis in myocardial cells by the activation of caspase-1 through activated STAT1 after focal ischemia and reperfusion damage (Gupta et al., 2011; Sun et al., 2011). Other studies have suggested the activation of STAT1 under focal ischemic or agglutination damage, as many strong antioxidants exert their protective function on cardiac cells and the brain by inhibiting STAT1 phosphorylation and nuclear entry (Kim et al., 2010). Apoptosis is a process of programmed cell death under the direction of apoptotic genes by specific activation of promoter regions. This process is different from necrotic cell death, which presents as severe cell damage by elevated membrane permeability, extension of the endoplasmic reticulum, and lysosome degradation, culminating in a severe inflammatory response. Apoptotic cells show a condensed nucleus, fragmented cytosolic inclusions, aggregation of the cytoplasm, and degraded DNA (Kang and Hébert, 2011). A complicated molecular mechanism underlies apoptosis, as hundreds of genes are directly involved in the initiation or inhibition of programmed cell death. Among them, caspase family, Bcl-2, and Bax genes have been extensively studied. Caspases regulate both apoptosis and proliferation/differentiation, and Bcl-2 and Bax have antagonistic expression patterns and effects on apoptosis (Yuan and He, 2013). The study of apoptosis has critical implications in studies of the pathogenesis of $\mathrm{SCl}$.

Based on previous studies, we hypothesized that STAT1 plays a role in SCI pathogenesis. We used STAT1 treatment and interference to observe its effects on SCI. An SCI rat model was first prepared according to established methods. After STAT1 or STAT1 inhibitor treatment, motor functions in both groups were evaluated and scored. The result indicated the alleviation of SClrelated motor dysfunction by treatment with an STAT1 inhibitor. We postulated related morphological alterations in spinal cord tissues corresponding to STAT1 or STAT1 inhibitor treatment. By H\&E staining, we found few neuronal cells and abundant vacuoles in the STAT1 treatment group, which is in contrast to larger number of neurons and few vacuoles in the STAT1 inhibitor-treated group. These results support our initial hypothesis that the STAT1 inhibitor could effectively alleviate $\mathrm{SCl}$-related tissue damage. To further investigate the underlying mechanism, we investigated the effect of STAT1 on apoptosis by showing the downregulation of the pro-apoptotic protein Bax and upregulation of the anti-apoptotic protein Bcl-2 in the STAT1 inhibitor treatment group. In addition, the TUNEL assay showed significant numbers of apoptotic cells in the STAT1-treated group but not in the STAT1 inhibitor-treated group. In summary, our results revealed the alleviation of SCI by the STAT1 inhibitor. This study supported previous findings that abnormal STAT1 expression is related to $\mathrm{SCI}$. It also showed the potency of the STAT1 inhibitor in reversing complications after the development of SCl. 


\section{Conflicts of interest}

The authors declare no conflict of interest.

\section{ACKNOWLEDGMENTS}

We thank the anonymous reviewers for reviewing this manuscript.

\section{REFERENCES}

Aittomäki S and Pesu M (2014). Therapeutic targeting of the Jak/STAT pathway. Basic Clin. Pharmacol. Toxicol. 114: 18-23. http://dx.doi.org/10.1111/bcpt.12164

Cao F, Hata R, Zhu P, Nakashiro K, et al. (2010). Conditional deletion of Stat3 promotes neurogenesis and inhibits astrogliogenesis in neural stem cells. Biochem. Biophys. Res. Commun. 394: 843-847.http://dx.doi.org/10.1016/j. bbrc.2010.03.092

Cao HQ and Dong ED (2013). An update on spinal cord injury research. Neurosci. Bull. 29: 94-102.http://dx.doi.org/10.1007/ $\underline{\mathrm{s} 12264-012-1277-8}$

Gómez-Nicola D, Valle-Argos B, Pallas-Bazarra N and Nieto-Sampedro M (2011). Interleukin-15 regulates proliferation and self-renewal of adult neural stem cells. Mol. Biol. Cell 22: 1960-1970.http://dx.doi.org/10.1091/mbc.E11-01-0053

Gupta S, Mishra K, Surolia A and Banerjee K (2011). Suppressor of cytokine signalling-6 promotes neurite outgrowth via JAK2/STAT5-mediated signalling pathway, involving negative feedback inhibition. PLoS One 6: e26674.http://dx.doi. org/10.1371/journal.pone.0026674

Han X, Lu M, Wang S, Lv D, et al. (2012). Targeting IKK/NF-кB pathway reduces infiltration of inflammatory cells and apoptosis after spinal cord injury in rats. Neurosci. Lett. 511: 28-32.http://dx.doi.org/10.1016/j.neulet.2012.01.030

Hua R, Shi J, Wang X, Yang J, et al. (2013). Analysis of the causes and types of traumatic spinal cord injury based on 561 cases in China from 2001 to 2010. Spinal Cord 51: 218-221.http://dx.doi.org/10.1038/sc.2012.133

Ishii H, Tanabe S, Ueno M, Kubo T, et al. (2013). ifn- $\gamma$-dependent secretion of IL-10 from Th1 cells and microglia/macrophages contributes to functional recovery after spinal cord injury. Cell Death Dis. 4: e710.http://dx.doi.org/10.1038/cddis.2013.234

Kang W and Hébert JM (2011). Signaling pathways in reactive astrocytes, a genetic perspective. Mol. Neurobiol. 43: $147-154$. http://dx.doi.org/10.1007/s12035-011-8163-7

Kim YH, Chung JI, Woo HG, Jung YS, et al. (2010). Differential regulation of proliferation and differentiation in neural precursor cells by the Jak pathway. Stem Cells 28: 1816-1828.http://dx.doi.org/10.1002/stem.511

Laurence A, Pesu M, Silvennoinen O and O'Shea J (2012). JAK kinases in health and disease: an update. Open Rheumatol. J. 6: 232-244.http://dx.doi.org/10.2174/1874312901206010232

Lu P, Graham L, Wang Y, Wu D, et al. (2014). Promotion of survival and differentiation of neural stem cells with fibrin and growth factor cocktails after severe spinal cord injury. J. Vis. Exp. 89: e50641.

O'Shea JJ, Holland SM and Staudt LM (2013). JAKs and STATs in immunity, immunodeficiency, and cancer. N. Engl. J. Med. 368: 161-170.http://dx.doi.org/10.1056/NEJMra1202117 PubMed

Oyinbo CA (2011). Secondary injury mechanisms in traumatic spinal cord injury: a nugget of this multiply cascade. Acta Neurobiol. Exp. (Warsz.) 71: 281-299.

Schmitt NC, Trivedi S and Ferris RL (2015). STAT1 Activation is enhanced by cisplatin and variably affected by EGFR inhibition in HNSCC cells. Mol. Cancer Ther. Published online July 3, 3015.

Sun F, Park KK, Belin S, Wang D, et al. (2011). Sustained axon regeneration induced by co-deletion of PTEN and SOCS3. Nature 480: 372-375.http://dx.doi.org/10.1038/nature10594

Vahedi G, Takahashi H, Nakayamada S, Sun HW, et al. (2012). STATs shape the active enhancer landscape of T cell populations. Cell 151: 981-993.http://dx.doi.org/10.1016/j.cell.2012.09.044

Vawda R and Fehlings MG (2013). Mesenchymal cells in the treatment of spinal cord injury: current \& future perspectives. Curr. Stem Cell Res. Ther. 8: 25-38.http://dx.doi.org/10.2174/1574888X11308010005

Yoshimura K, Ueno M, Lee S, Nakamura Y, et al. (2011). c-Jun N-terminal kinase induces axonal degeneration and limits motor recovery after spinal cord injury in mice. Neurosci. Res. 71: 266-277.http://dx.doi.org/10.1016/j.neures.2011.07.1830

Yuan YM and He C (2013). The glial scar in spinal cord injury and repair. Neurosci. Bull. 29: 421-435.http://dx.doi.org/10.1007/ $\underline{\mathrm{s} 12264-013-1358-3}$ 